Individual raw values of diaphragm activity and MEP dia (average of 5 to 10 single MEP) 


\section{Sham animals}

Rat \#52

Ipsi

Diaphragm activity

MEP dia
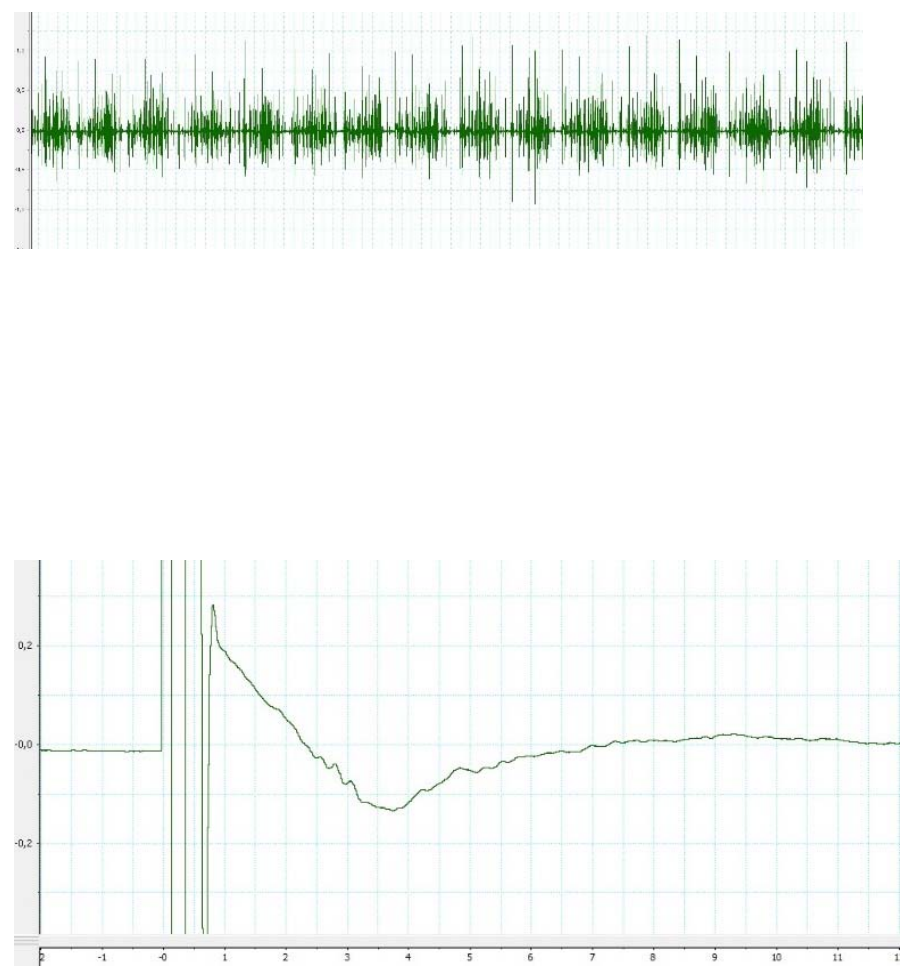

Contra

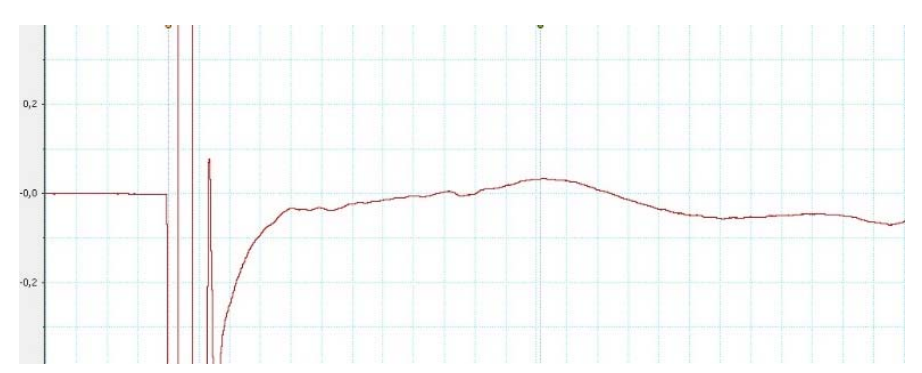




\section{Sham animals}

\section{Rat \#53}

Ipsi

Contra

Diaphragm activity

MEP dia
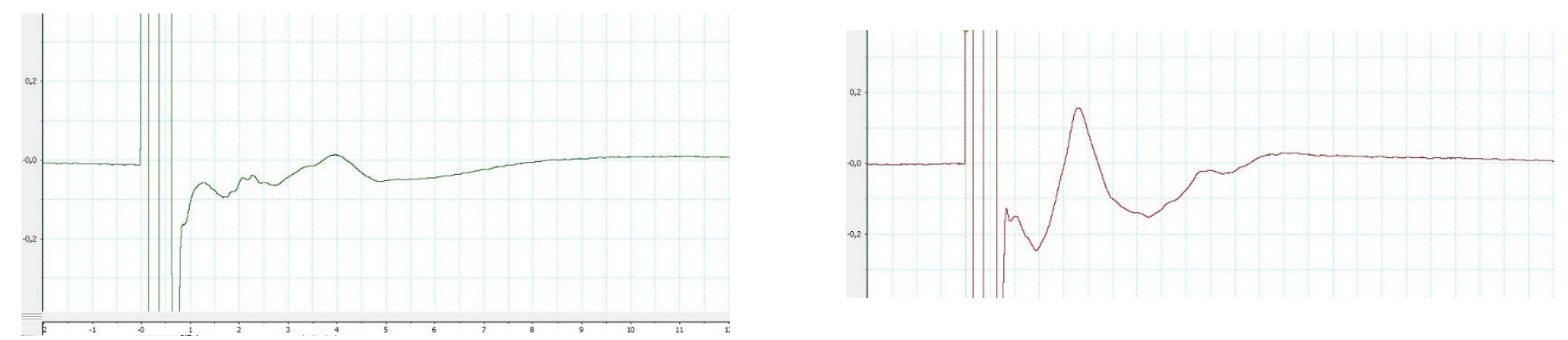


\section{Sham animals}

\section{Rat \#54}

$$
\text { Ipsi }
$$

Diaphragm activity

\section{MEP dia}
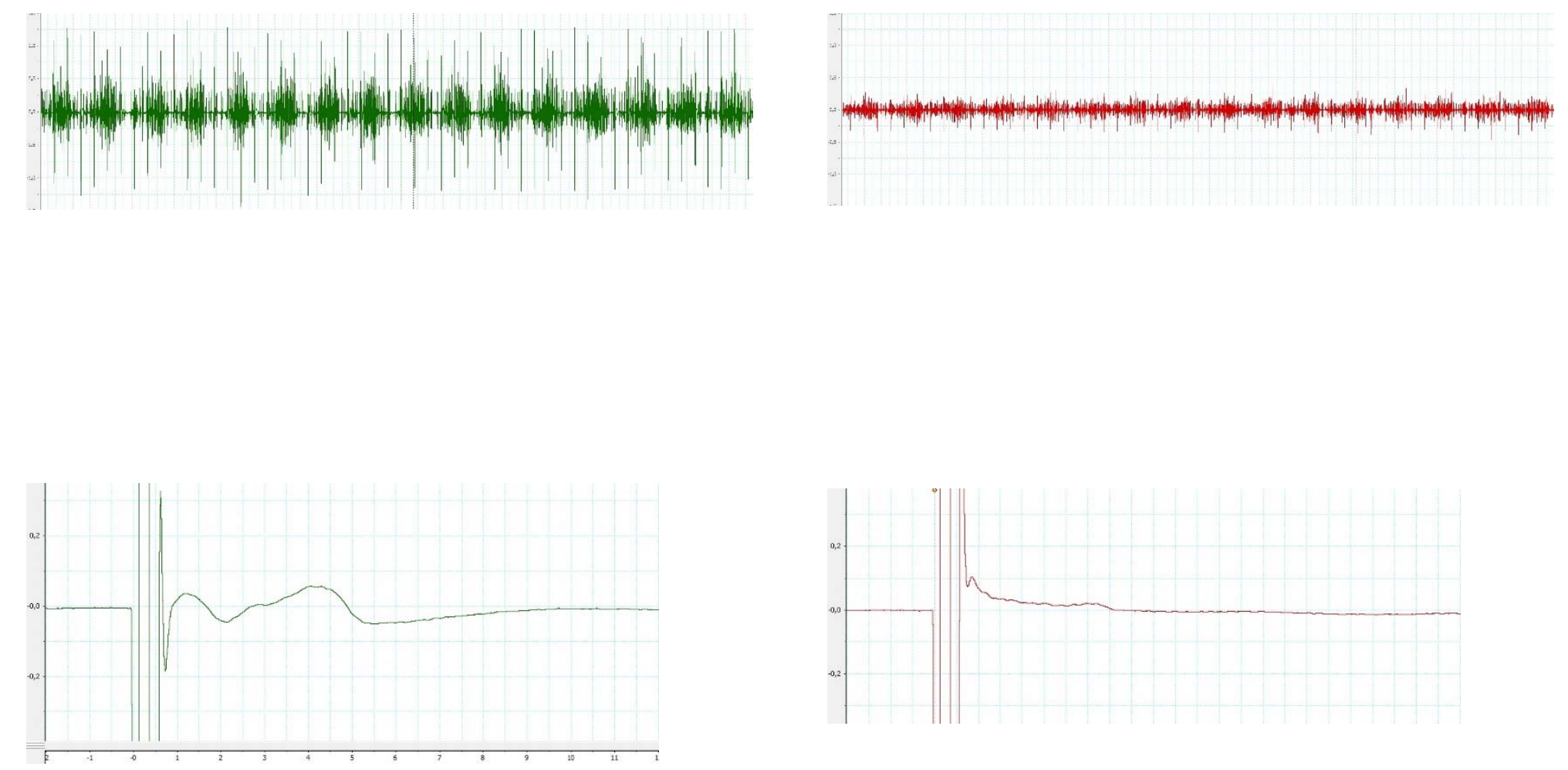

Contra

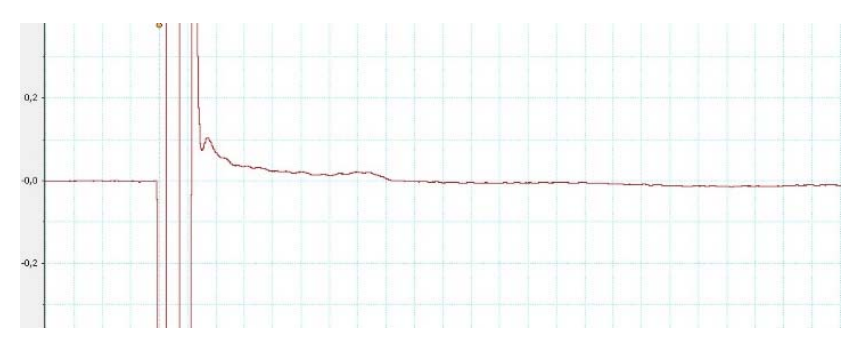




\section{Sham animals}

\section{Rat \#56}

Ipsi

Diaphragm activity

MEP dia
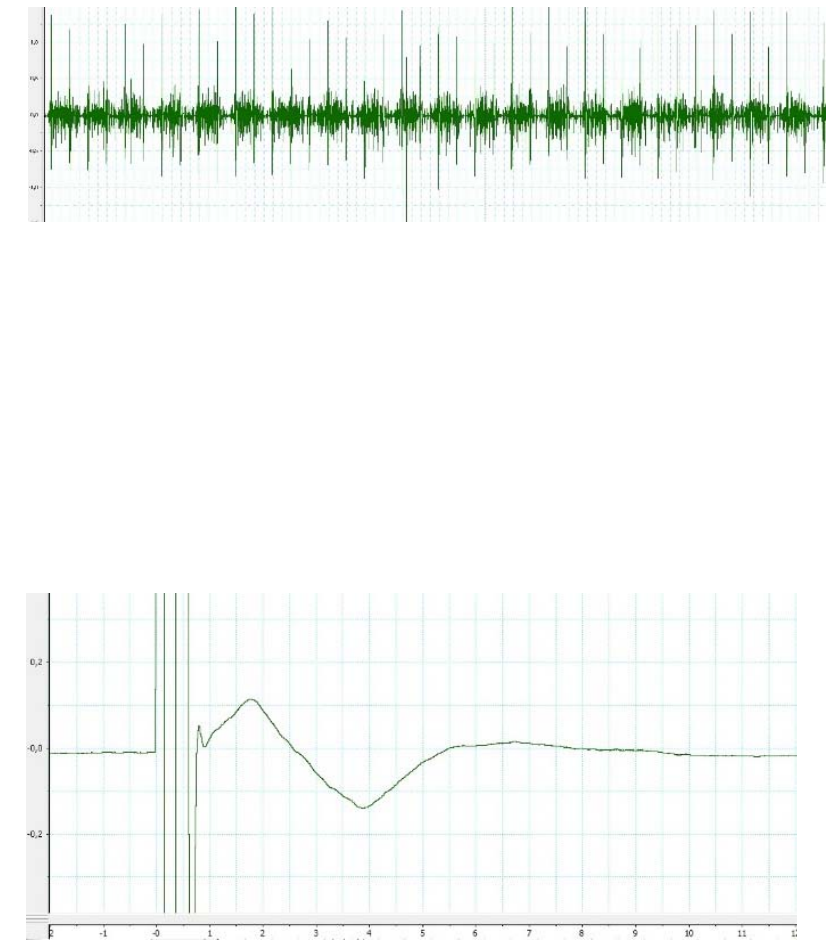

Contra
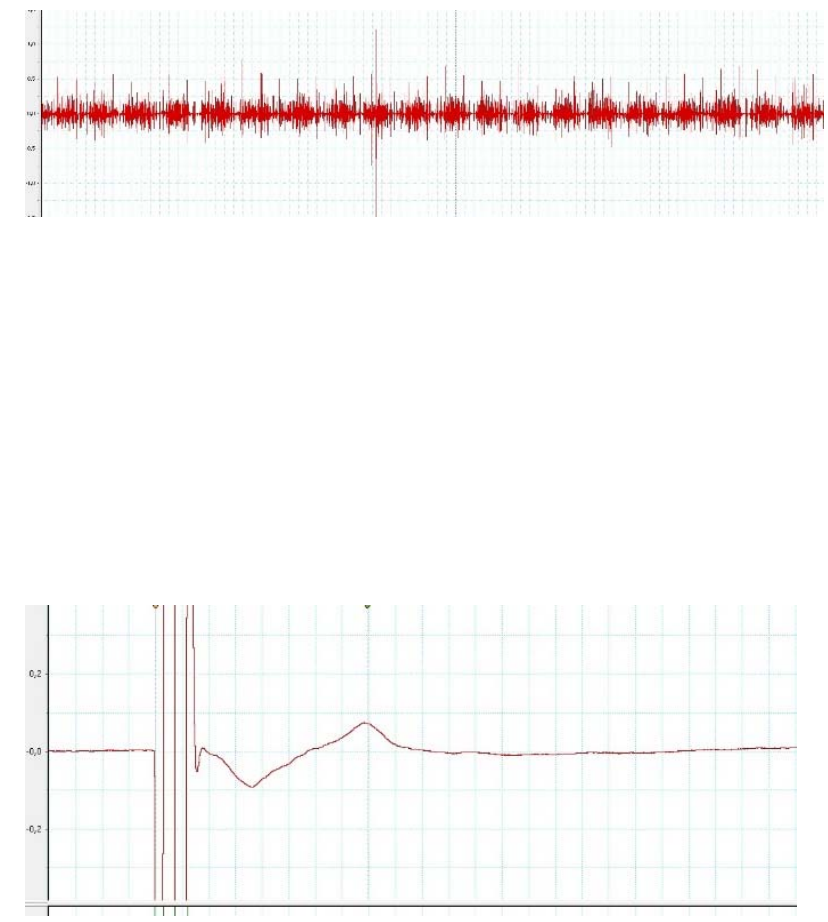


\section{Sham animals}

\section{Rat \#57}

Ipsi

Diaphragm activity

MEP dia

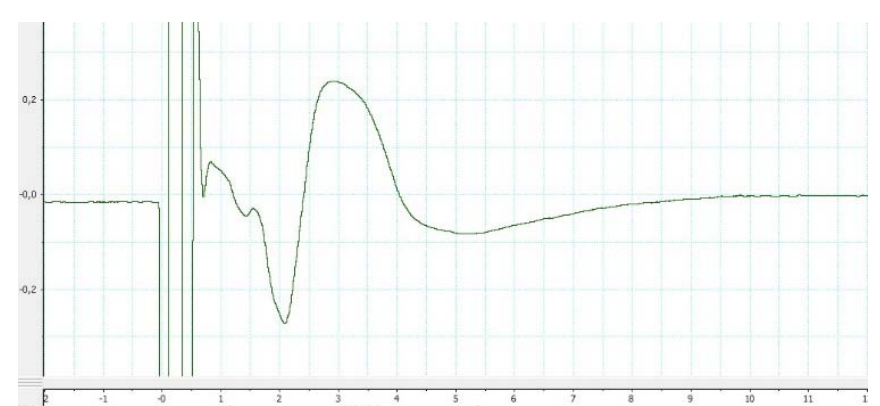

Contra

Whow Tham

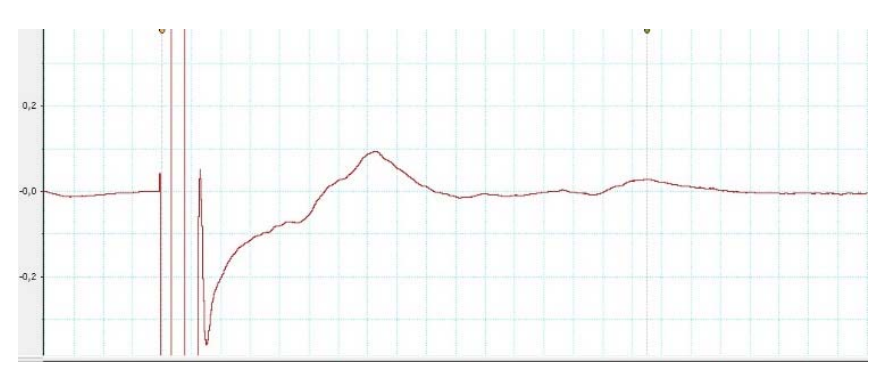


Sham animals

Rat \#58

Ipsi

Diaphragm activity

MEP dia
Contra

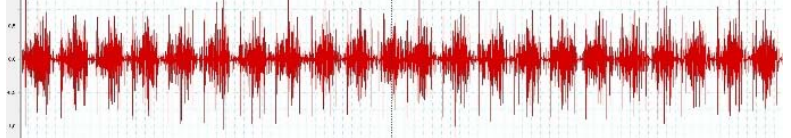

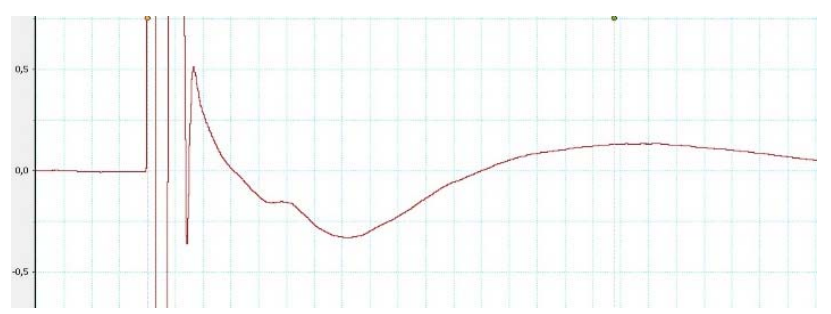




\section{Sham animals}

Rat \#59

Ipsi

Diaphragm activity

MEP dia
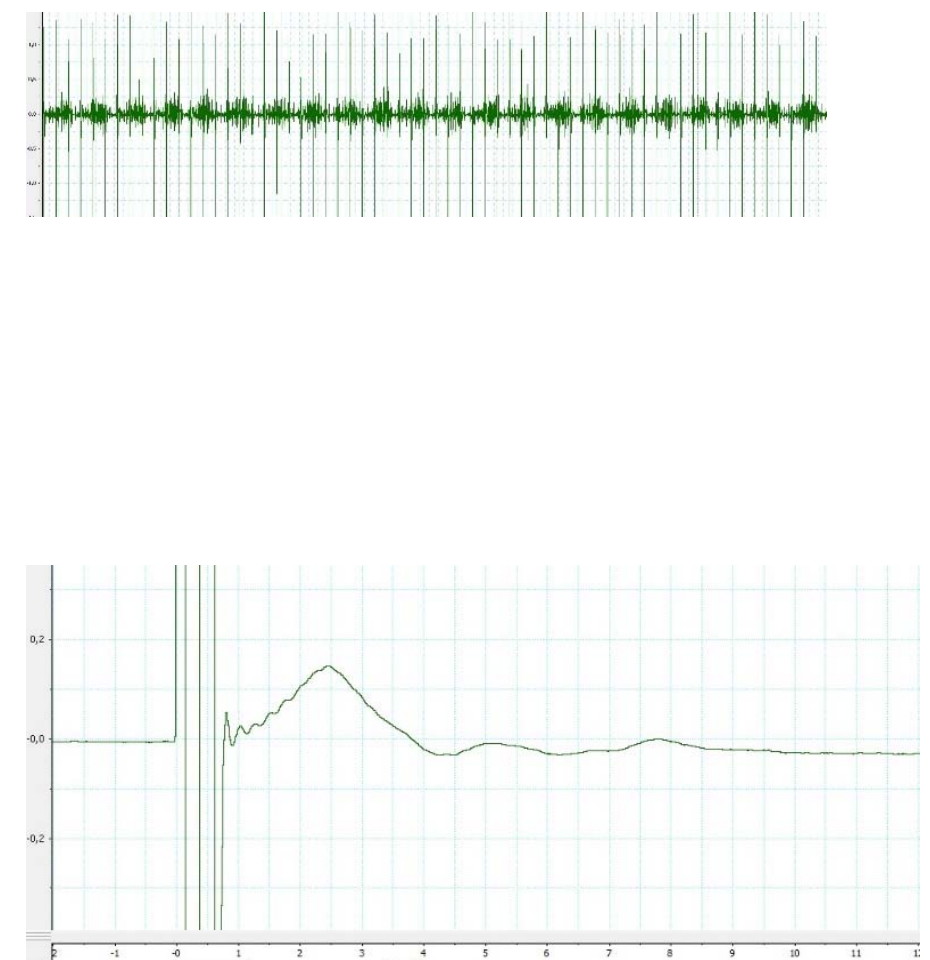

Contra

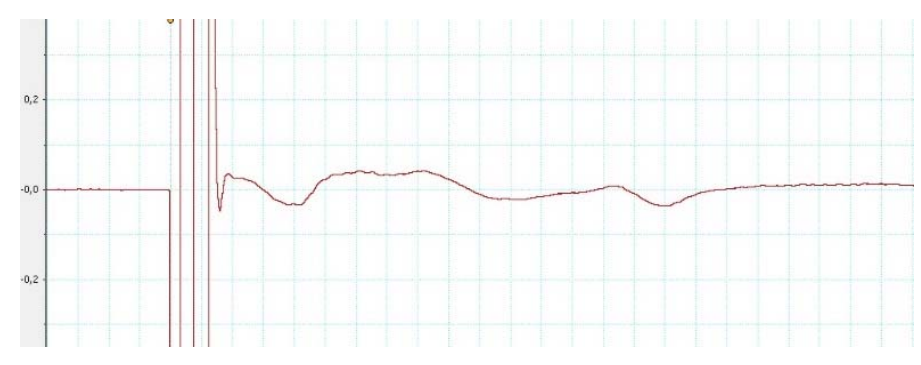




\section{Sham animals}

Rat \#60

Ipsi

Diaphragm activity

MEP dia
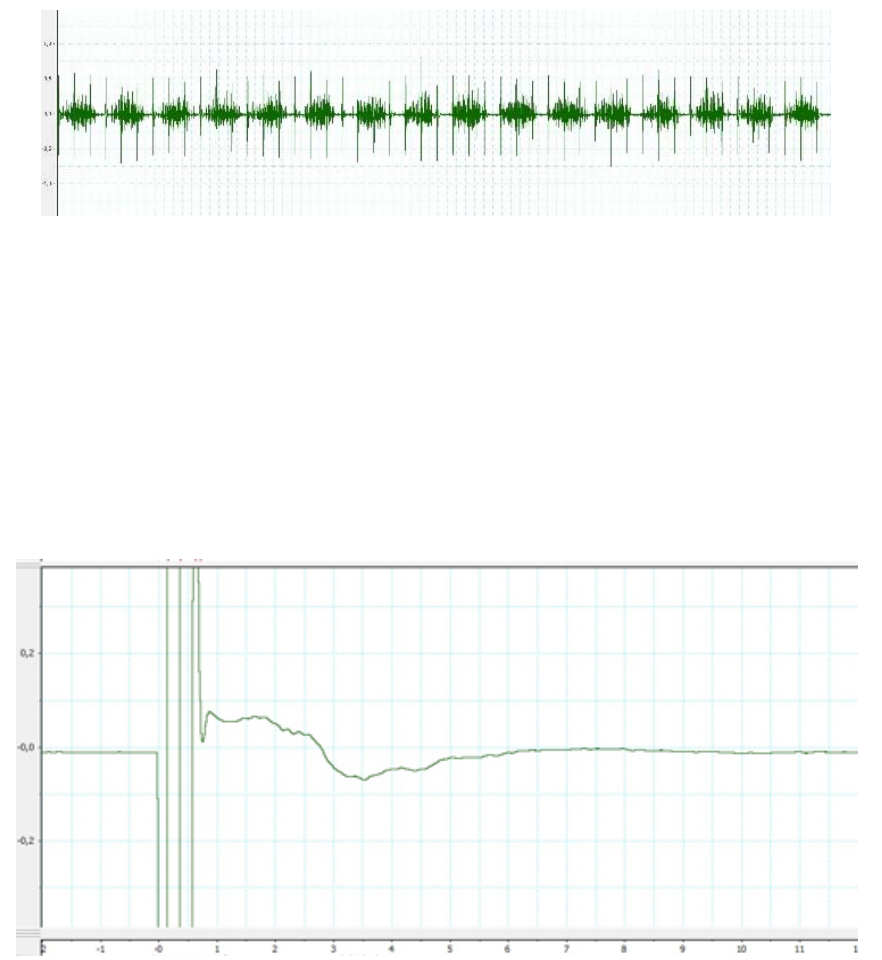

Contra

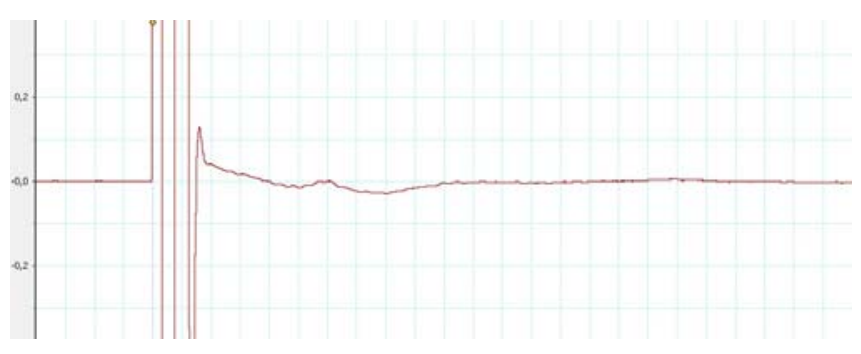




\section{Sham animals}

\section{Rat \#61}

Ipsi

Contra

Diaphragm activity
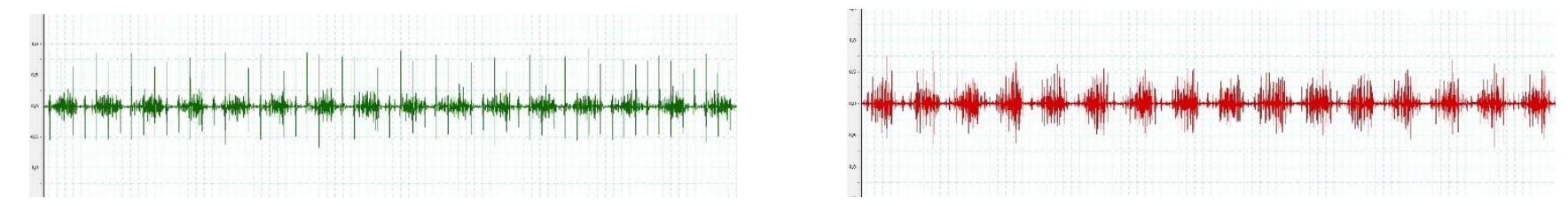

MEP dia
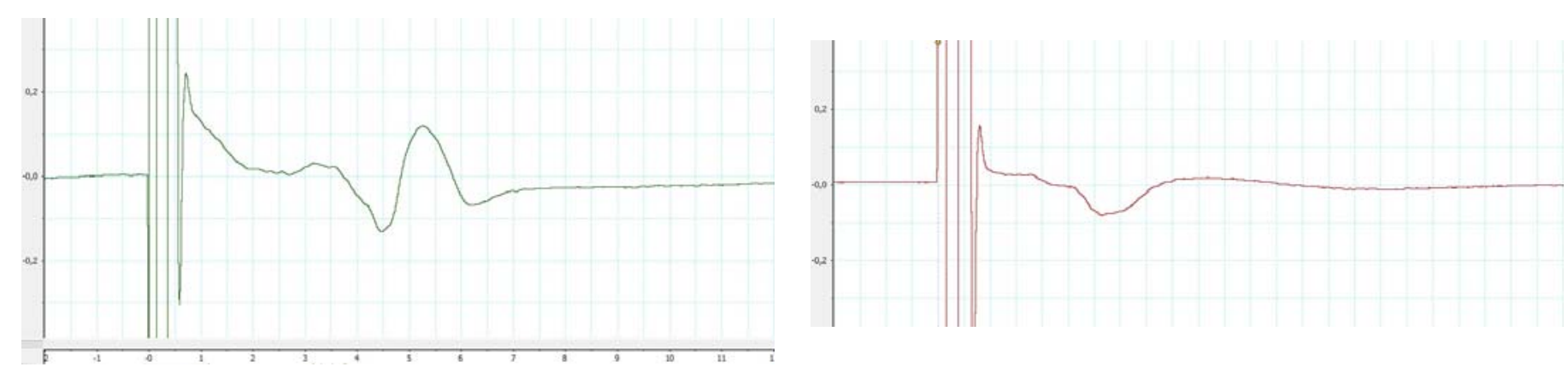


\section{Sham animals}

Rat \#62

Ipsi

Diaphragm activity

MEP dia
Contra

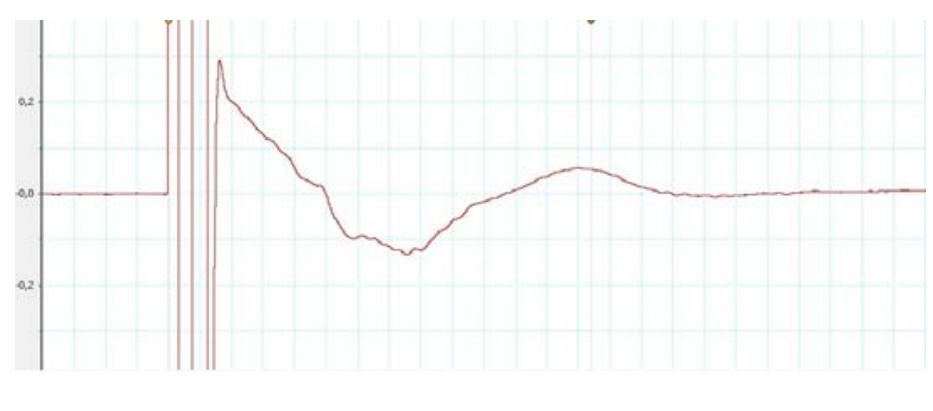




\section{Sham animals}

\section{Rat \#63}

Ipsi

Diaphragm activity

MEP dia
Contra
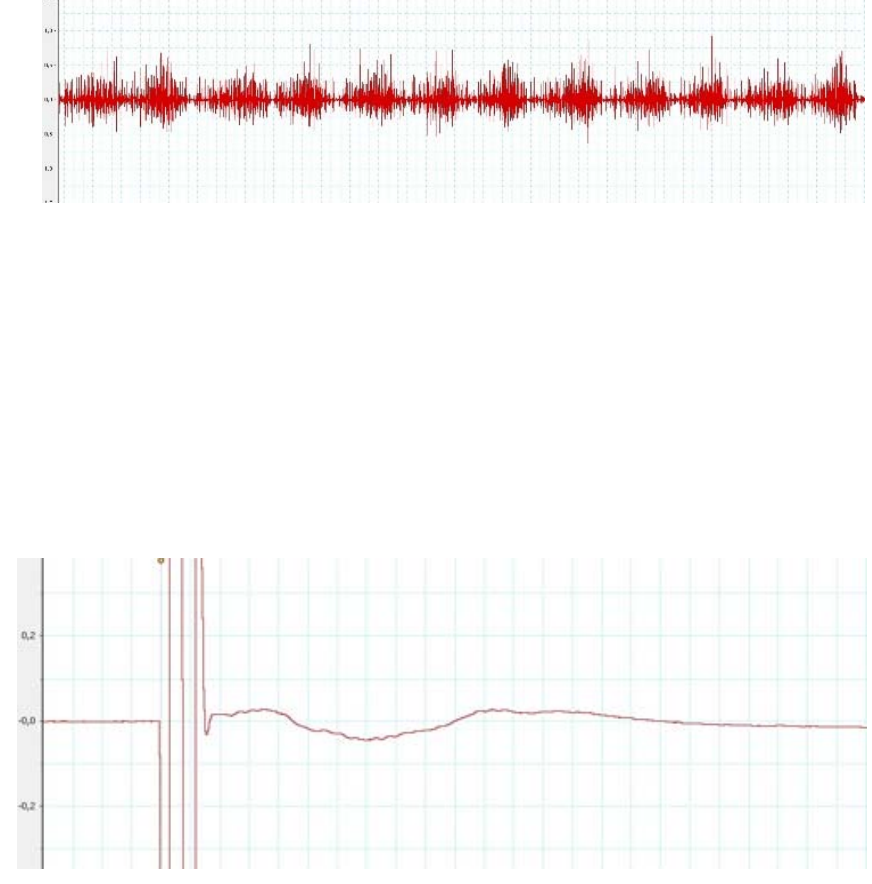


\section{Sham animals}

\section{Rat \#64}

Ipsi

Diaphragm activity

MEP dia
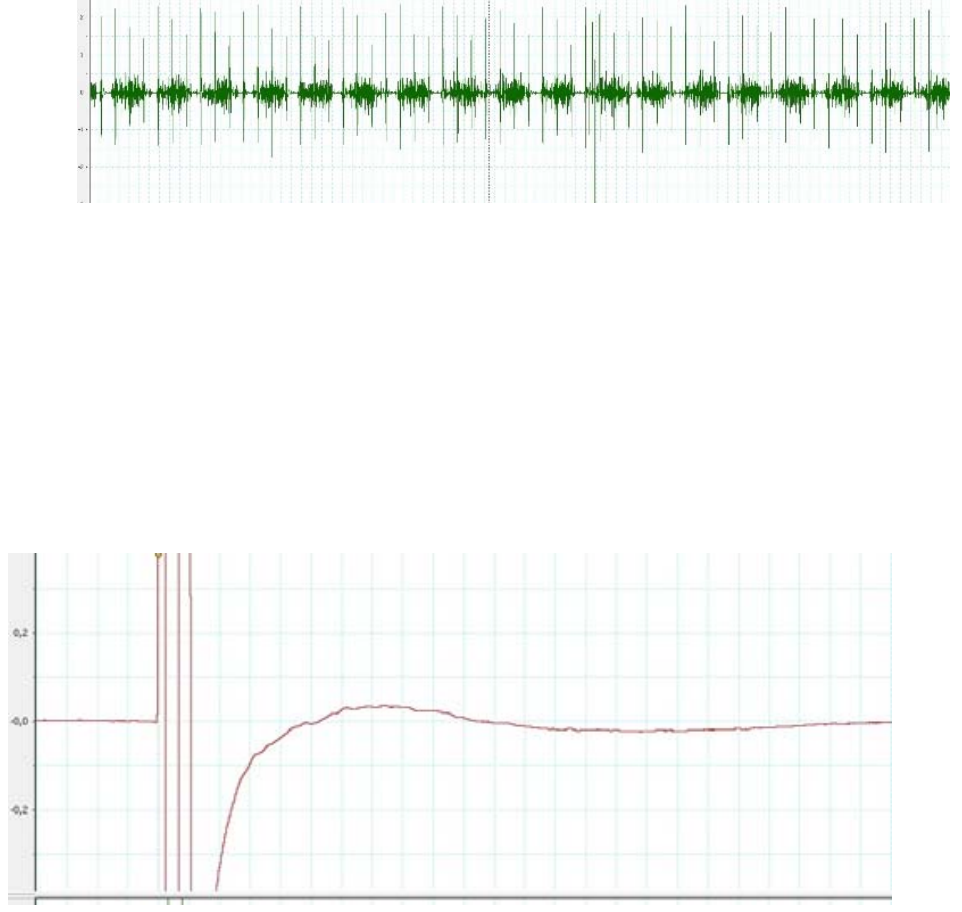

Contra

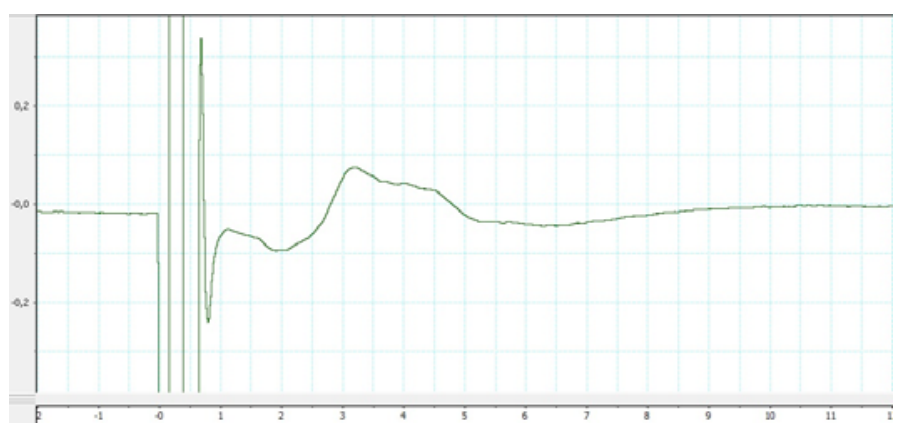




\section{Sham animals}

Rat \#65

Ipsi

Diaphragm activity

MEP dia
- य

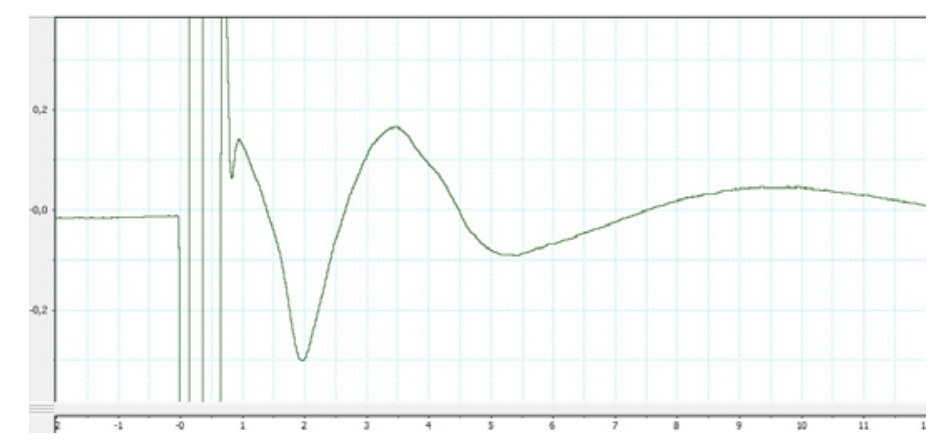

Contra

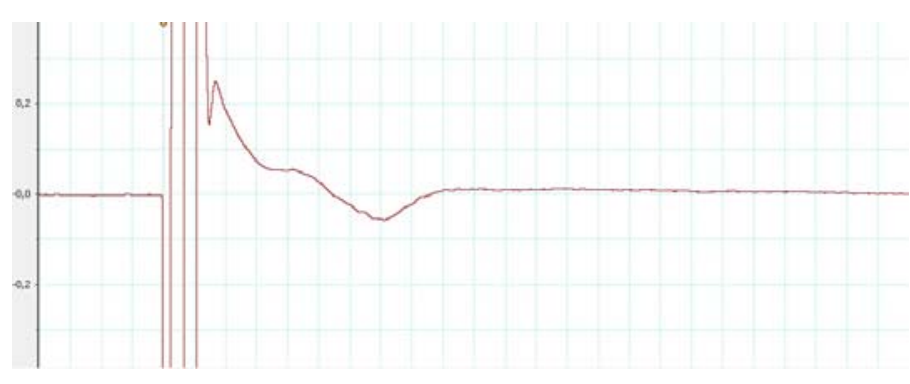




\section{Sham animals}

\section{Rat \#42}

Ipsi

Diaphragm activity

MEP dia
Contra

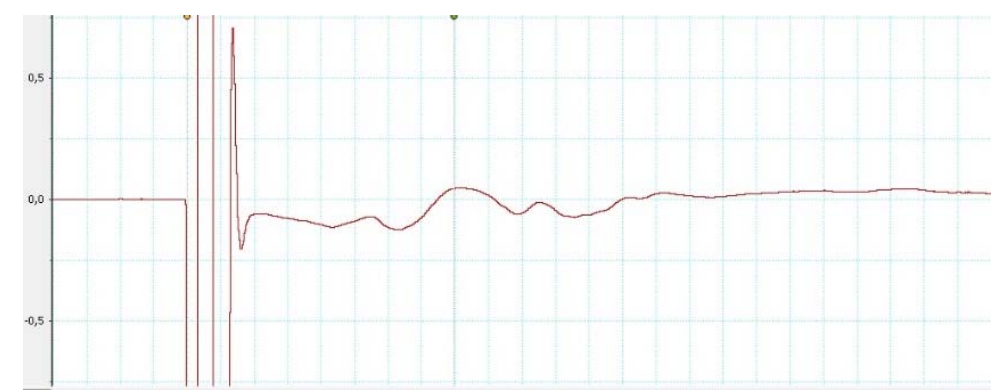




\section{Sham animals}

Rat \#41

Ipsi

Diaphragm activity

MEP dia
Contra
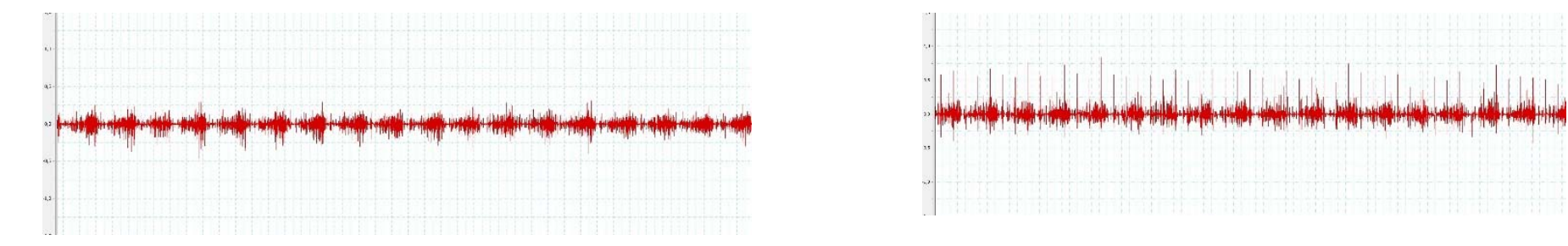

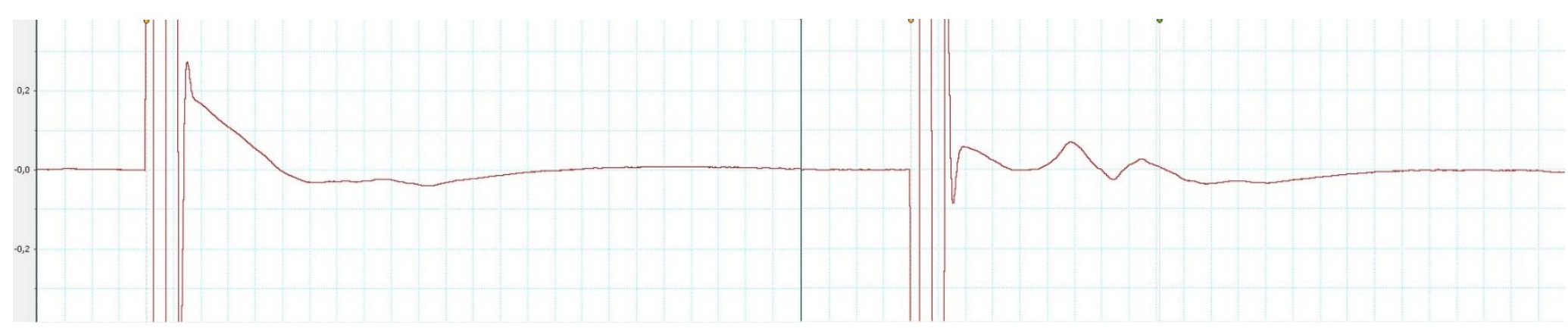




\section{Sham animals}

\section{Rat \#40}

Ipsi

Diaphragm activity

MEP dia
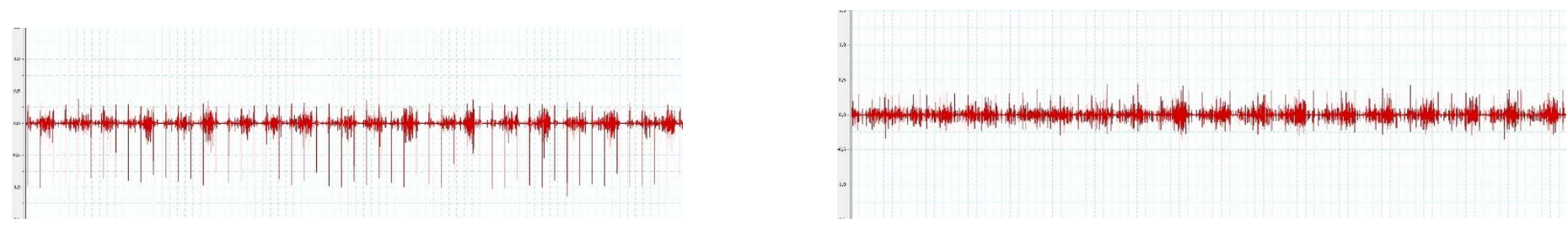

Contra

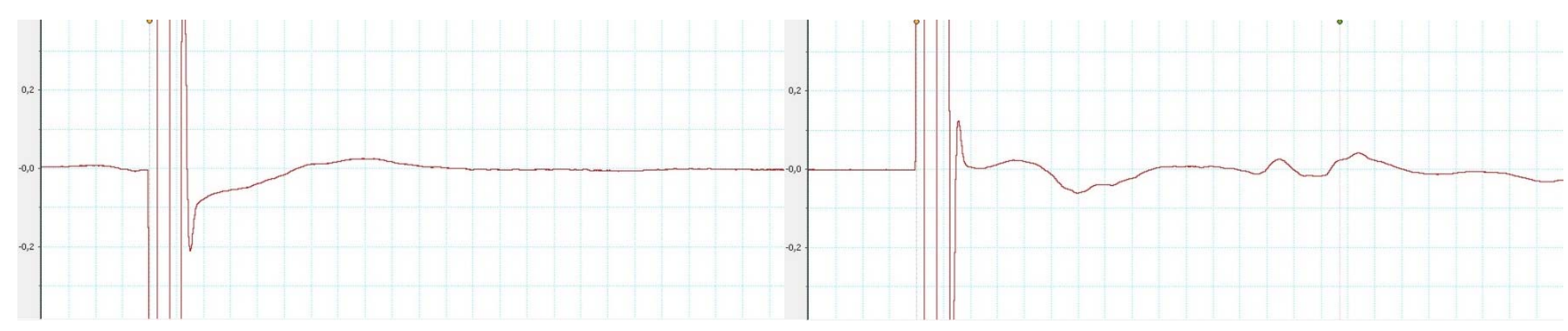




\section{Sham animals}

Rat \#72

Ipsi

Contra

Diaphragm activity

MEP dia

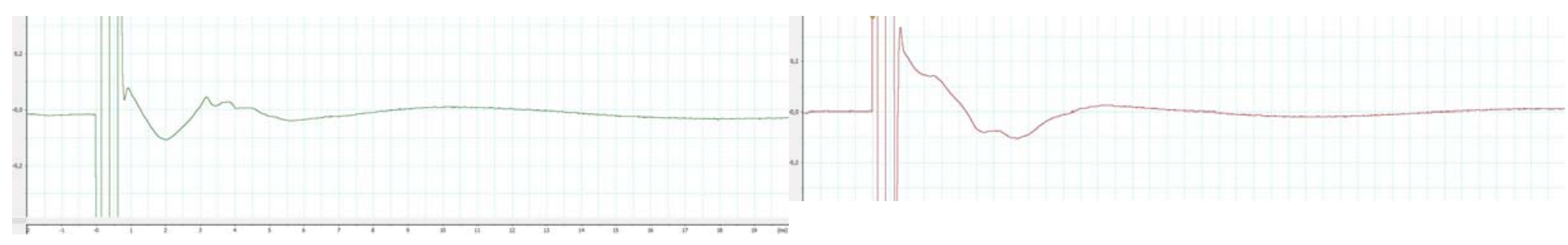




\section{Sham animals}

Rat \#71

Ipsi

Contra

Diaphragm activity

MEP dia
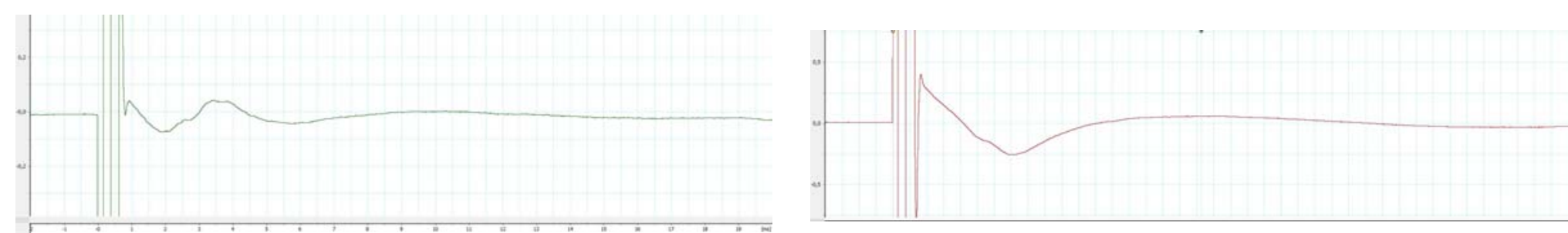
Sham animals

Rat \#66

Ipsi

Diaphragm activity

MEP dia
Contra

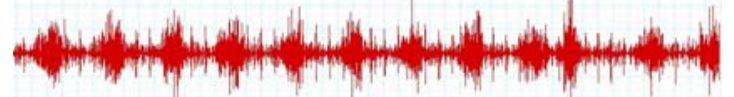




\section{Sham animals}

\section{Rat \#67}

Ipsi

Contra

Diaphragm activity

MEP dia
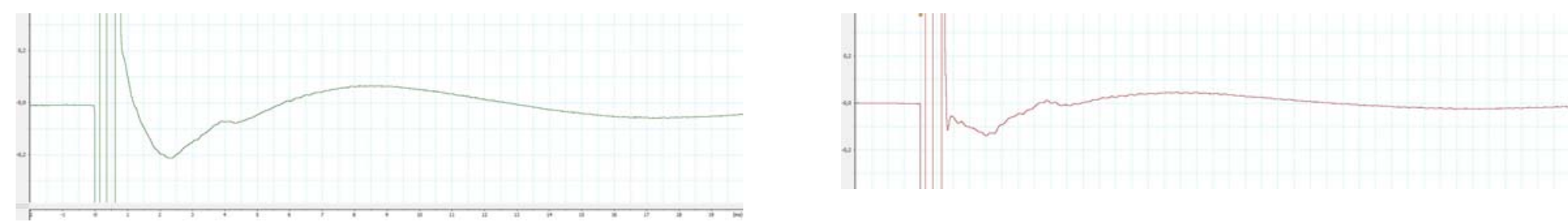


\section{Sham animals}

\section{Rat \#68}

Ipsi

Diaphragm activity

MEP dia
Contra

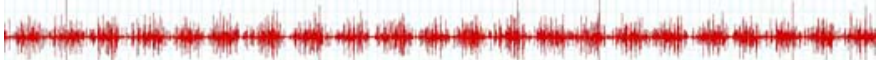
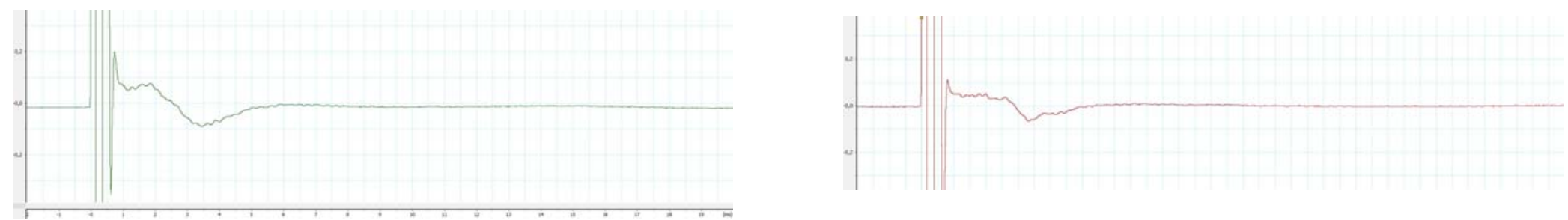
1h P.I.

Rat \#27

Ipsi

Contra

Diaphragm activity

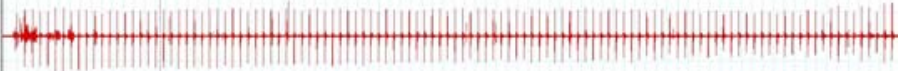

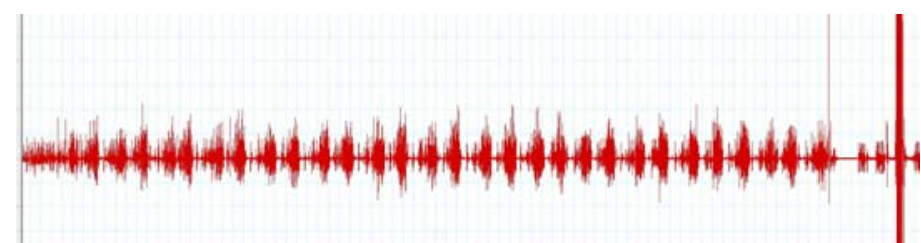

MEP dia
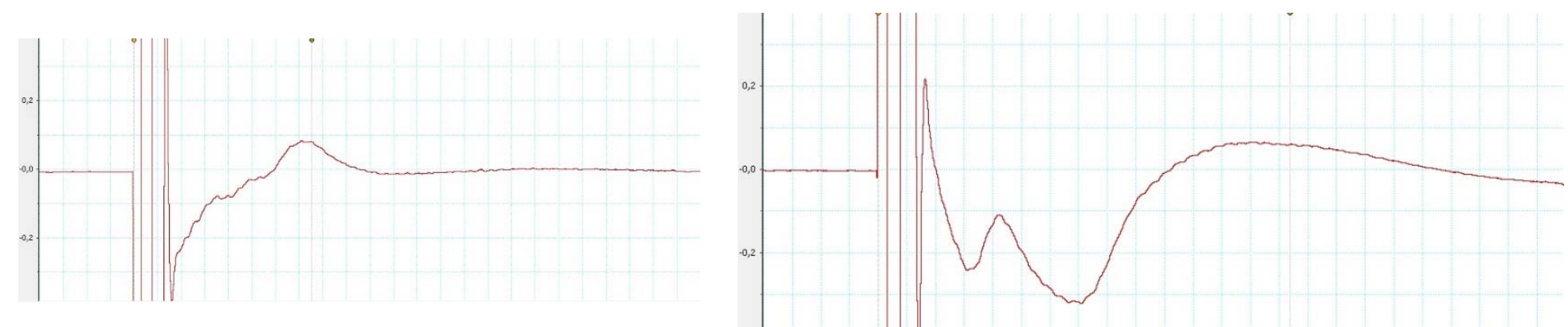
1h P.I.

Rat \#29

Ipsi

Diaphragm activity

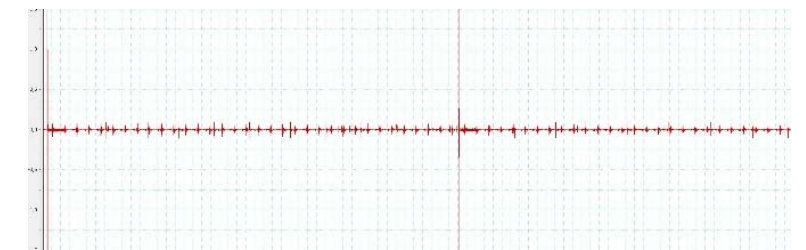

MEP dia
Contra

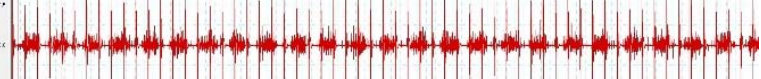
|| 
1h P.I.

Rat \#30

Ipsi

Diaphragm activity

MEP dia
Contra

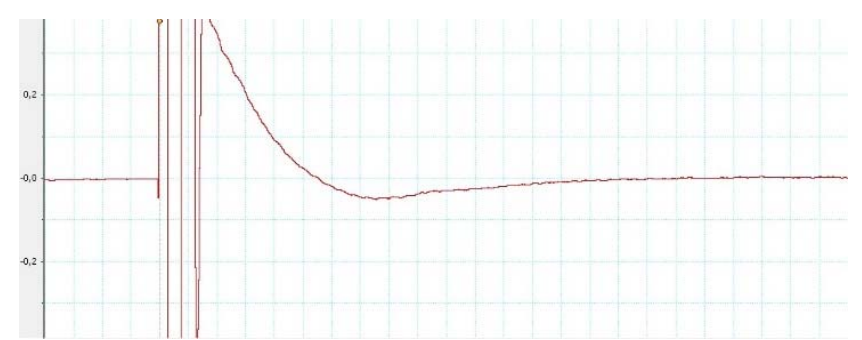


1h P.I.

Rat \#31

Ipsi

Diaphragm activity

MEP dia
Contra
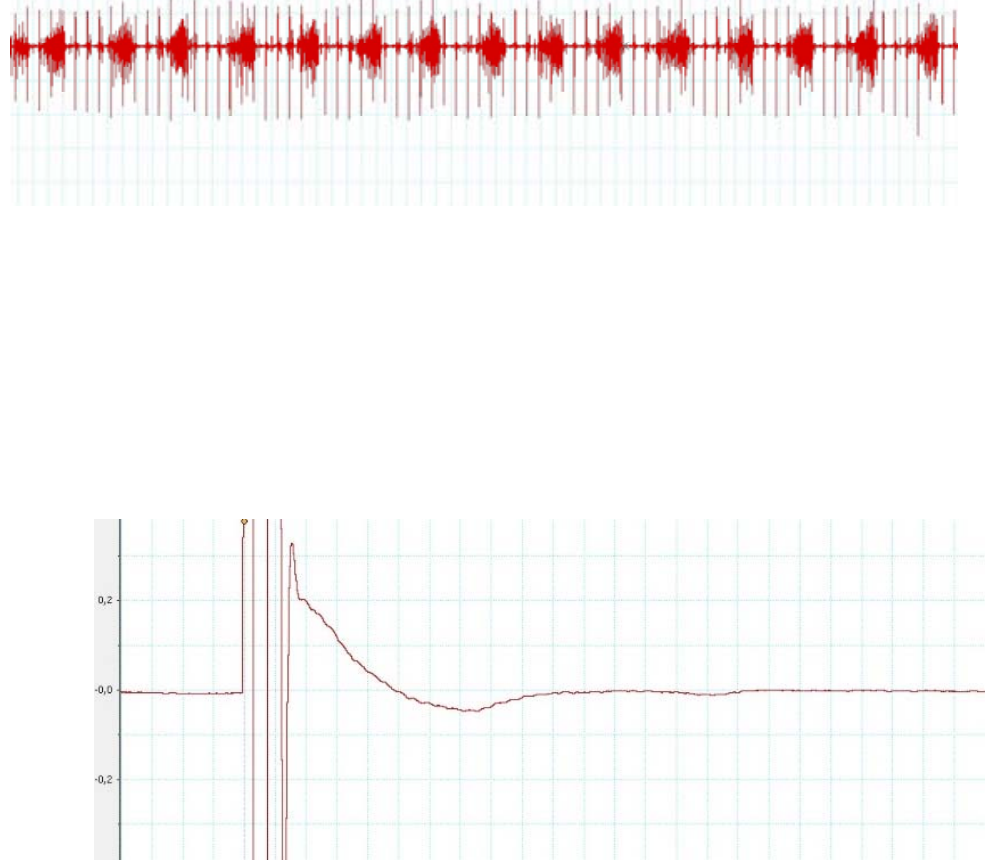
1h P.I.

Rat \#32

Ipsi

Diaphragm activity

MEP dia
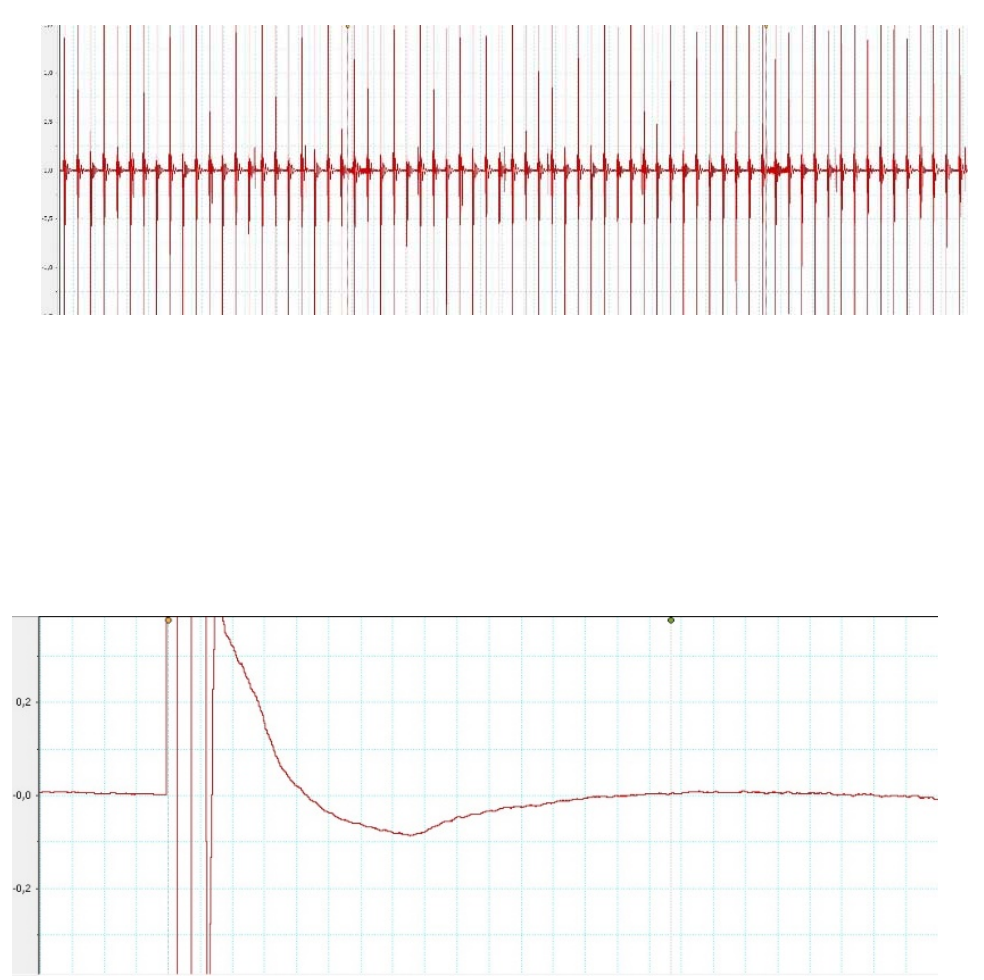

Contra

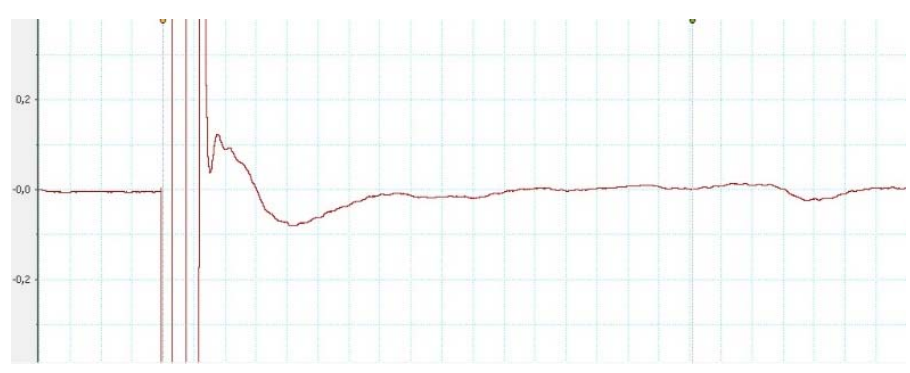


1h P.I.

Rat \#33

Ipsi

Diaphragm activity

MEP dia
Contra
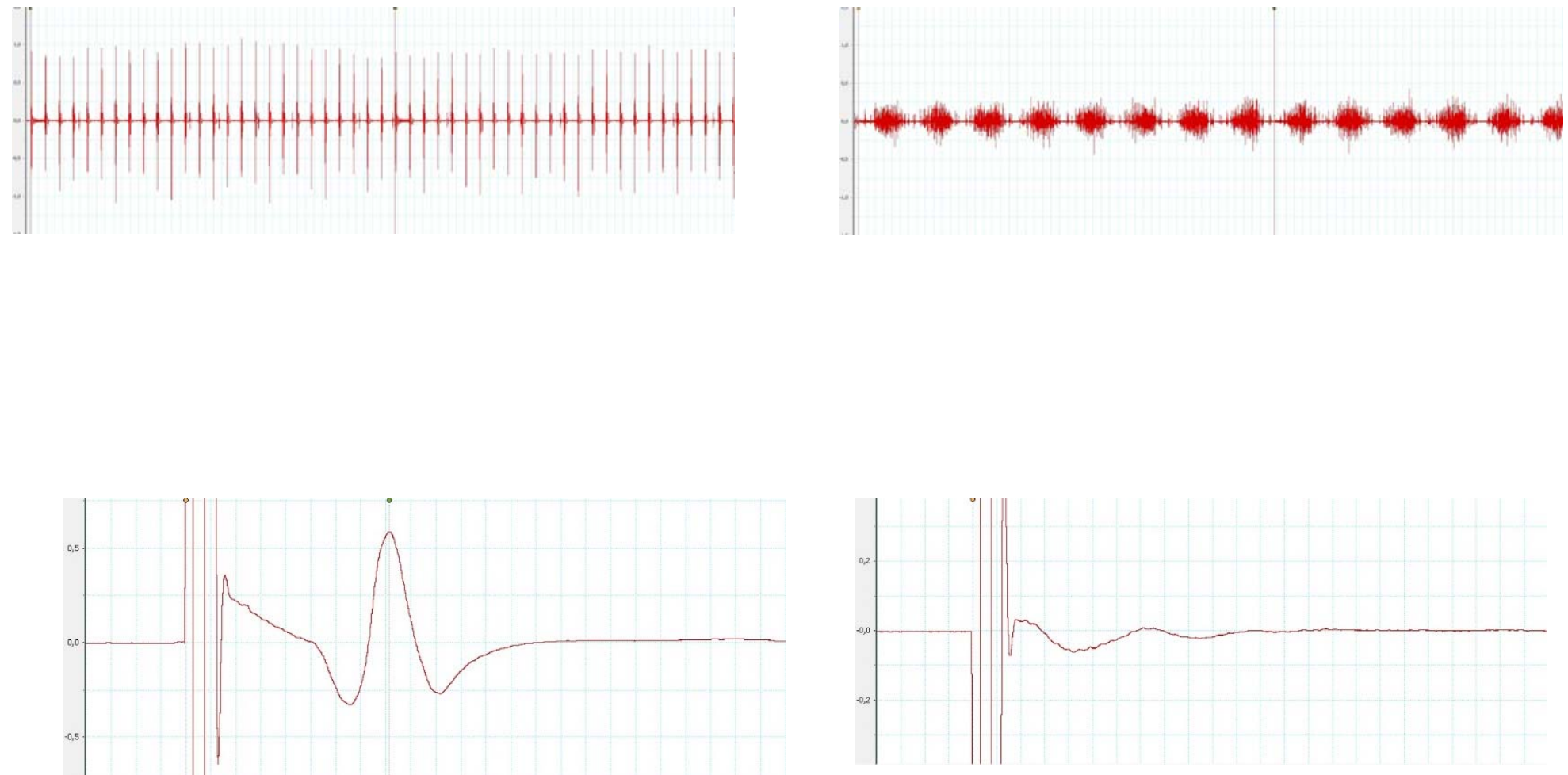
1h P.I.

Rat \#69

Ipsi

Diaphragm activity

MEP dia
Contra

$4+4+4+4+4+4+4+4+4+4+4+4+4$ 
1h P.I.

Rat \#70

Ipsi

Diaphragm activity

MEP dia
Contra

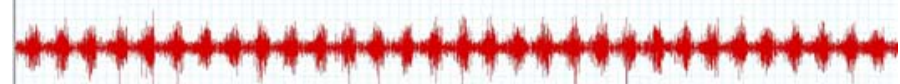

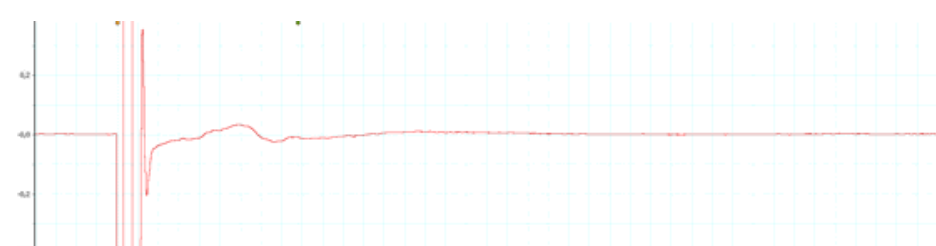


1h P.I.

Rat \#73

Ipsi

Diaphragm activity

MEP dia
Contra

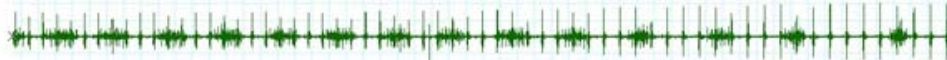


1h P.I.

Rat \#74

Diaphragm activity

MEP dia
Ipsi

Contra

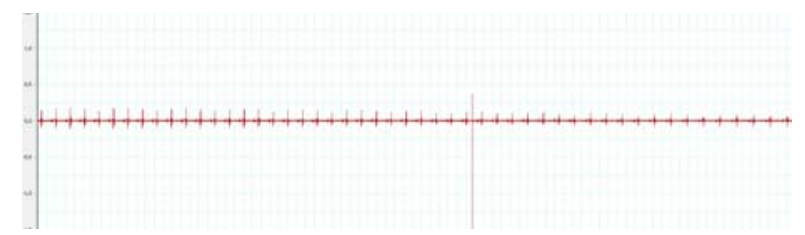

MTH 


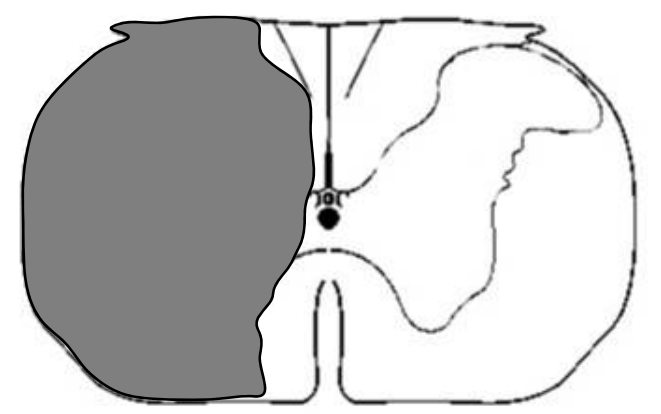

Ipsi

Diaphragm activity

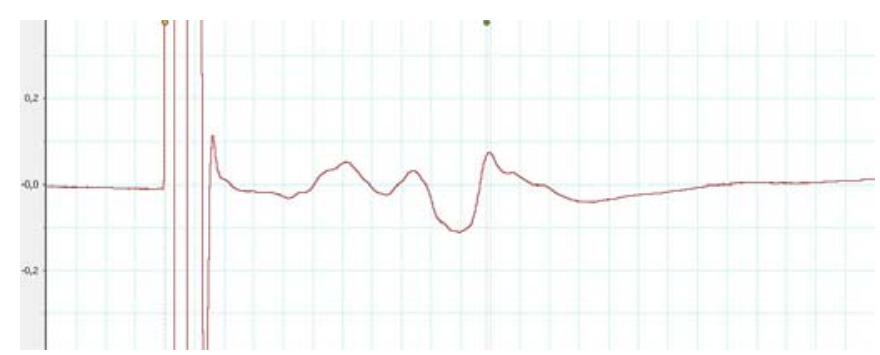

Contra

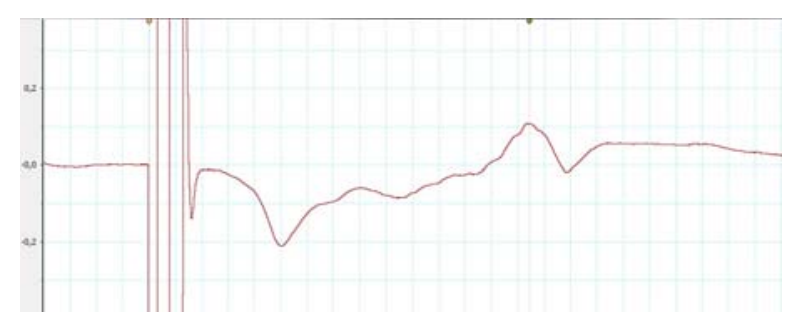




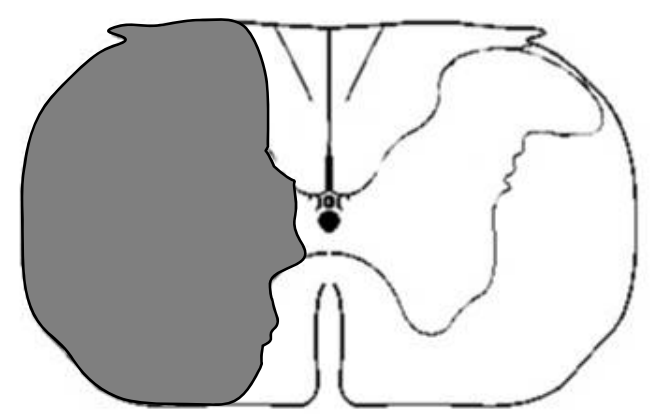

Ipsi

Diaphragm activity

MEP dia
7 d P.I.

Rat \#38

Contra

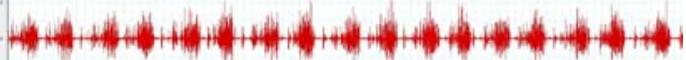

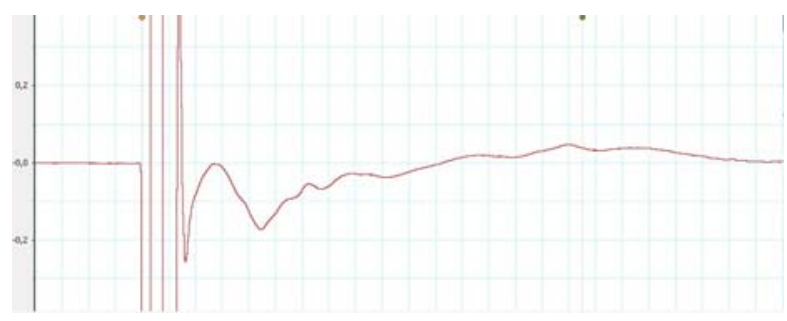




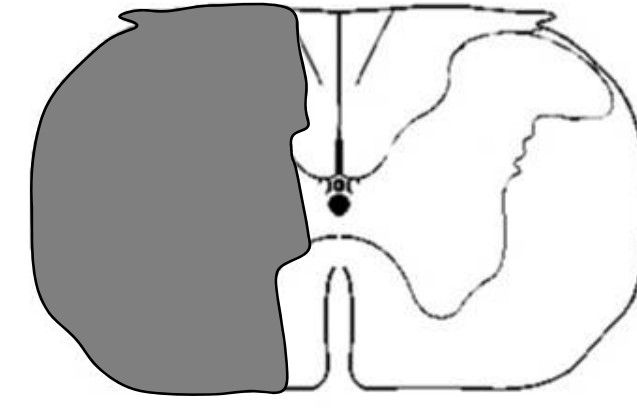

Ipsi

Diaphragm activity

MEP dia
7 d P.I.

Rat \#39

Contra

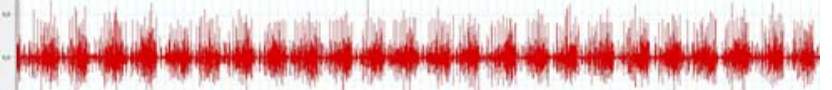
.

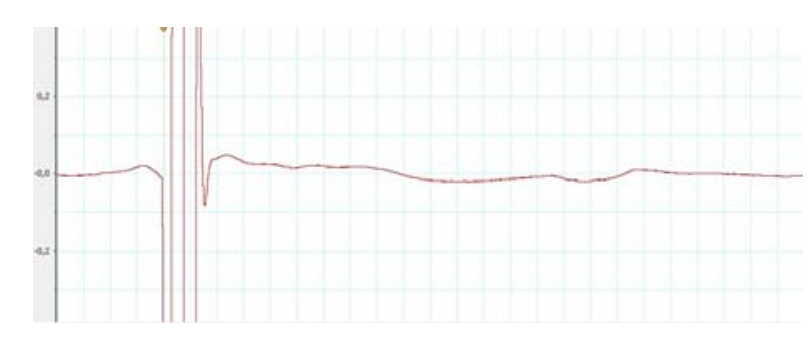




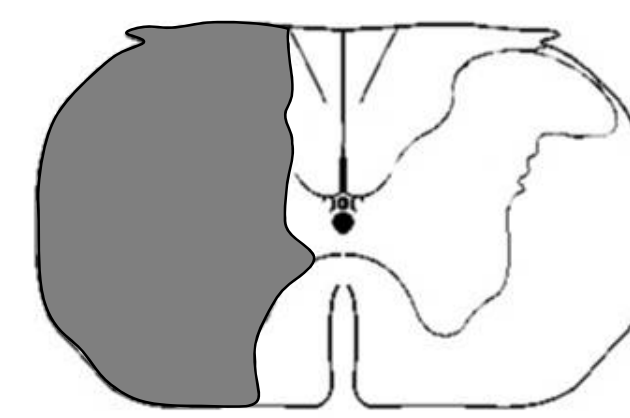

Diaphragm activity

Ipsi

MEP dia
7 d P.I.

Rat \#43
Contra

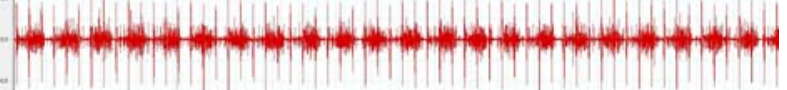




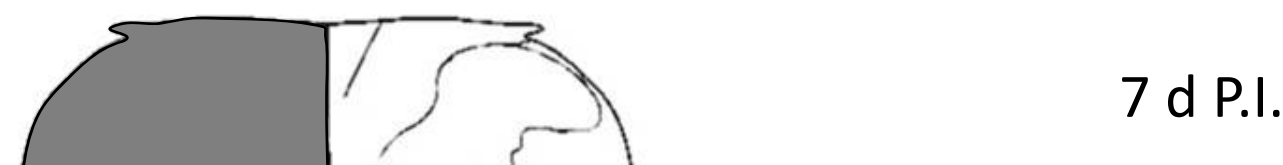

Rat \#46

Contra

Diaphragm activity

MEP dia
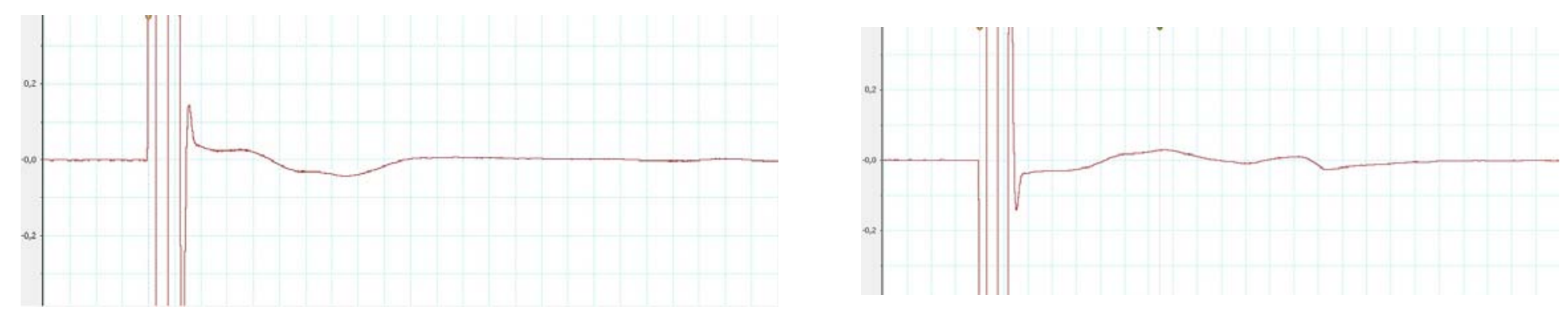


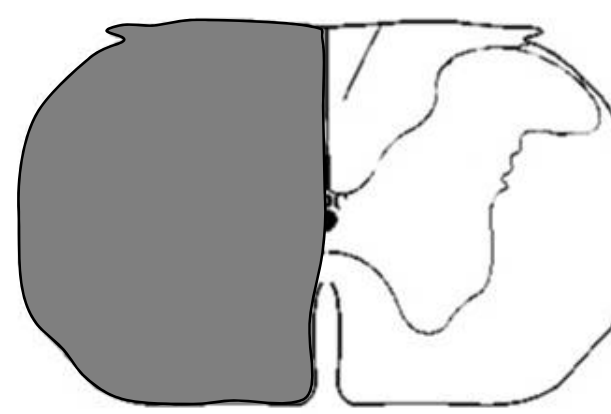

Ipsi

Diaphragm activity

MEP dia
7 d P.I.

Rat \#47

Contra
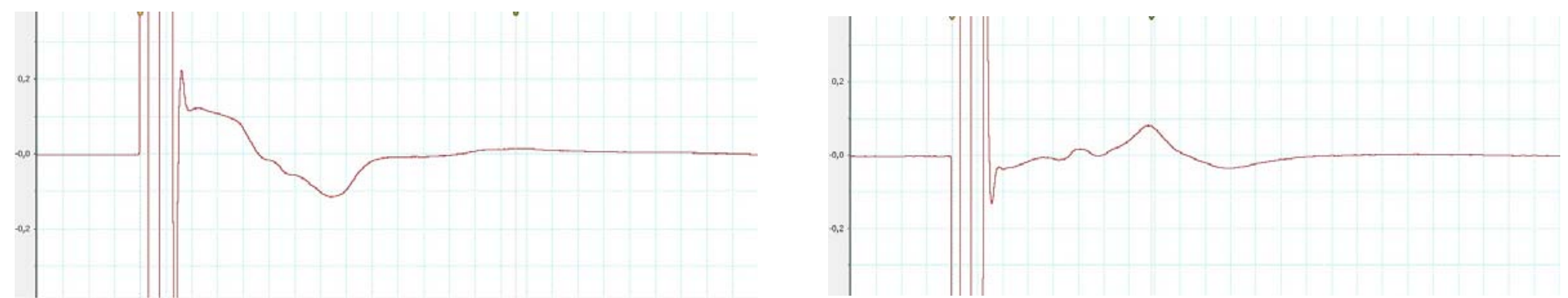


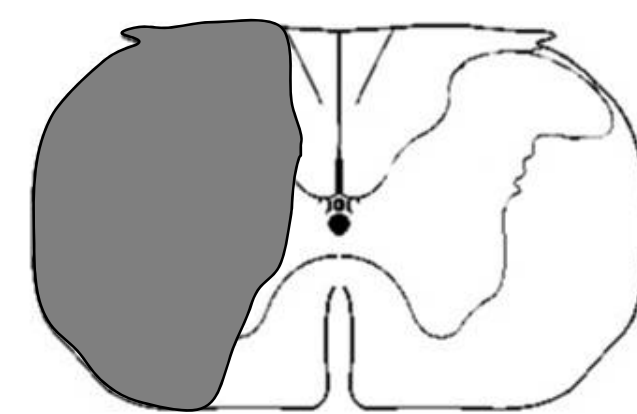

Ipsi

Diaphragm activity

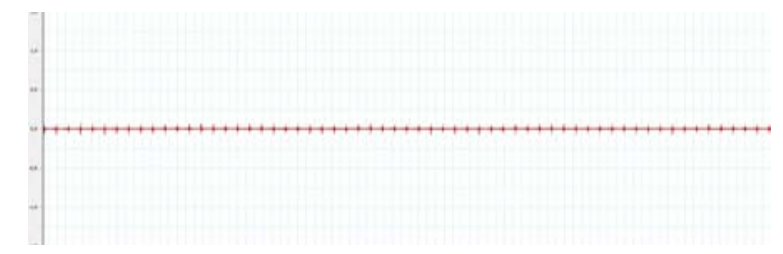

MEP dia
7 d P.I.

Rat \#50

\section{Contra}
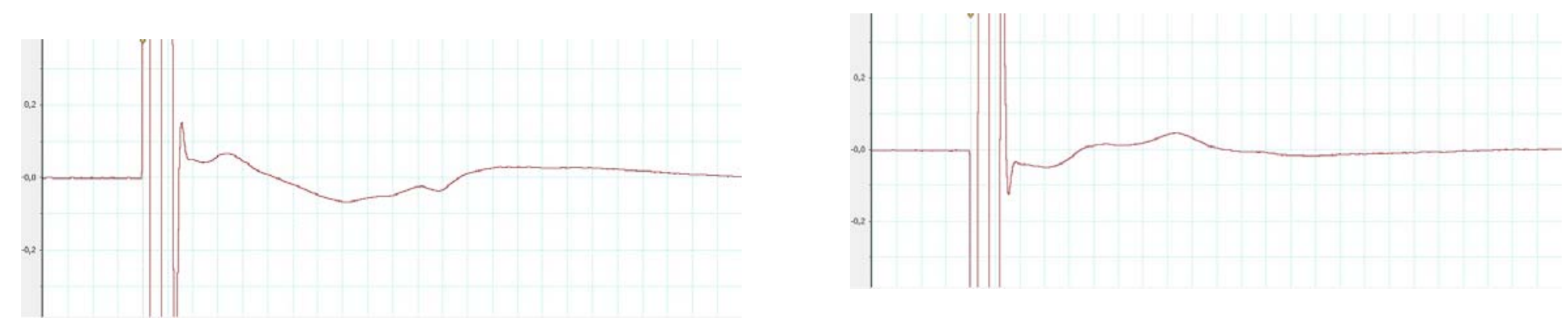


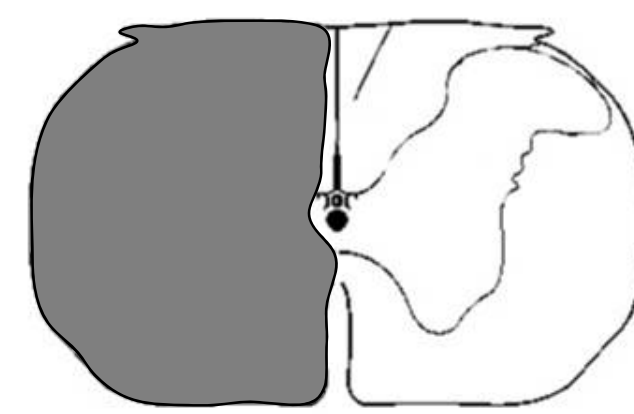

Ipsi

Diaphragm activity
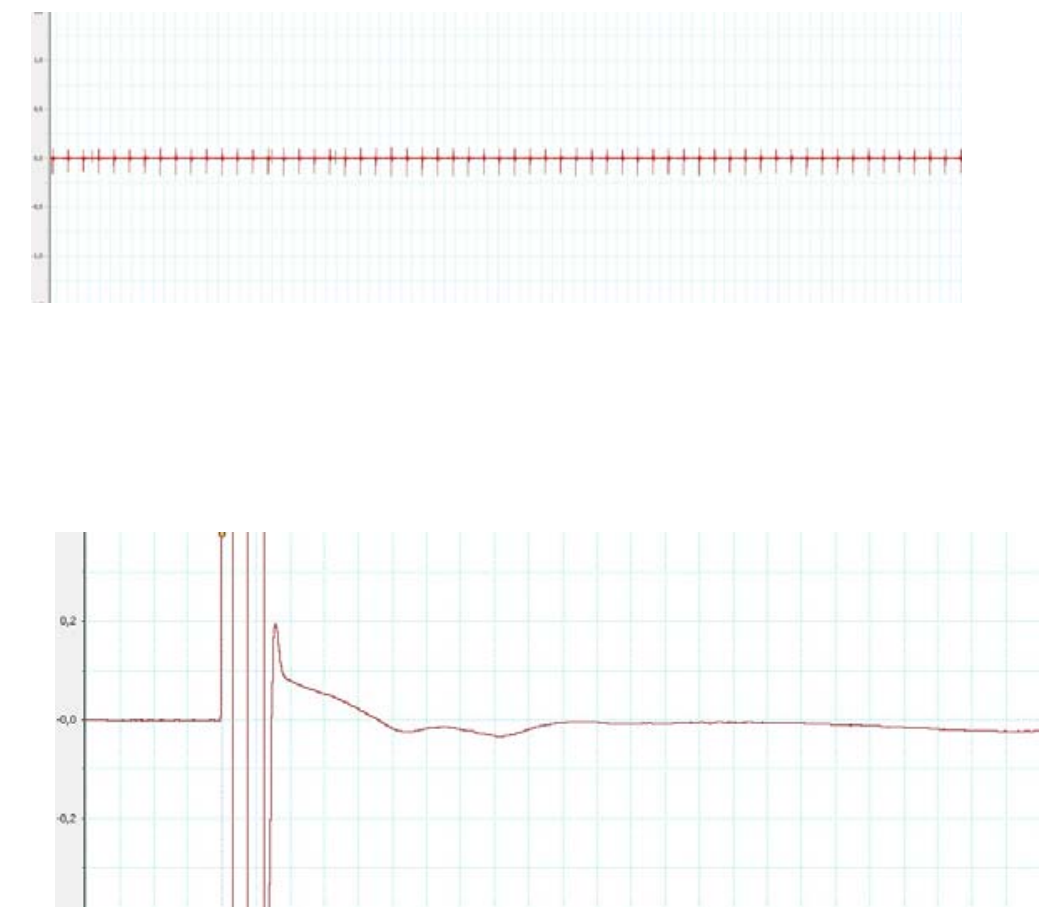

MEP dia
Contra

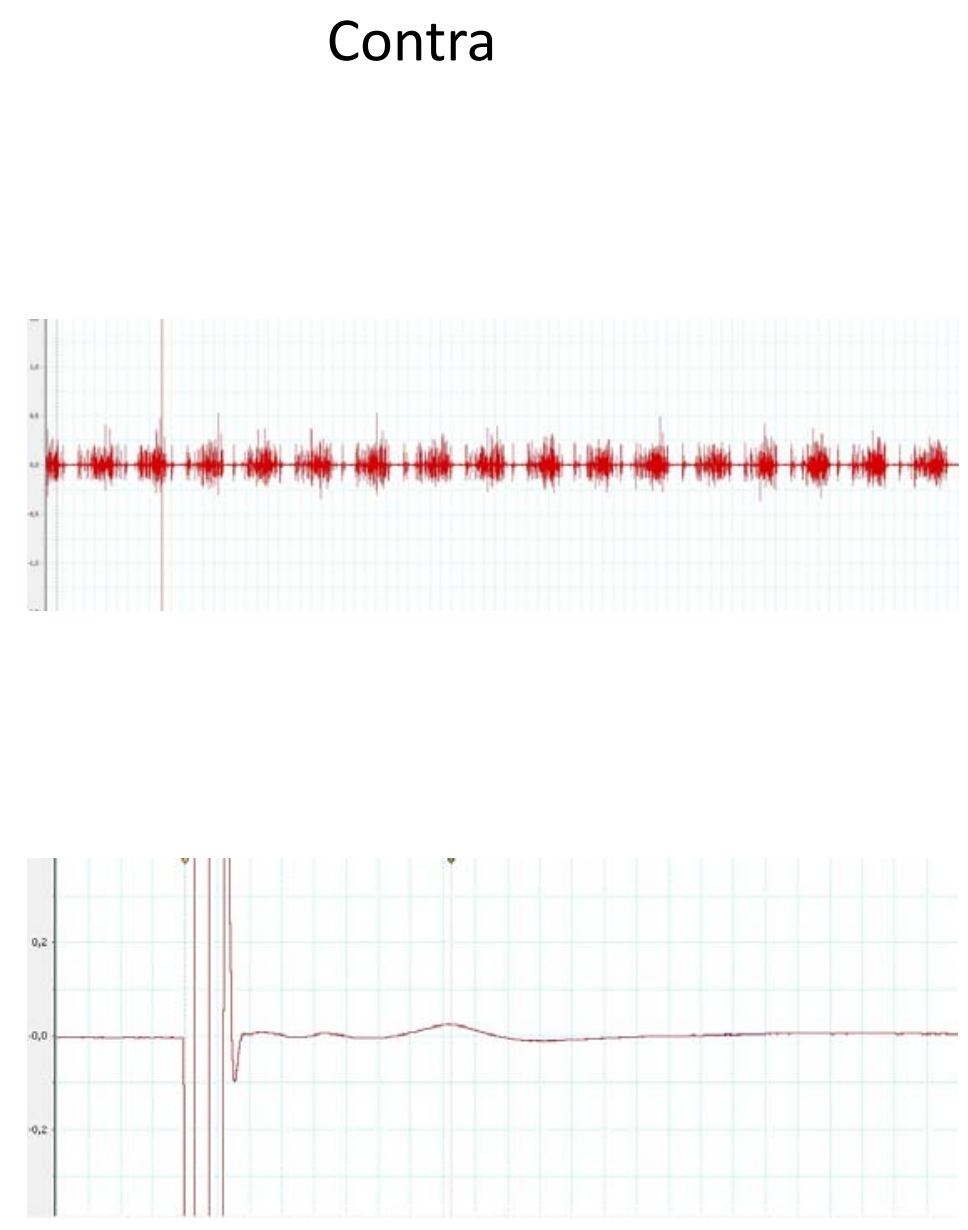

7 d P.I.

Rat \#51 